\title{
SUBDIRECT PRODUCTS OF SEMIRINGS
}

\section{P. MUKHOPADHYAY}

(Received 19 July 1999)

\begin{abstract}
Bandelt and Petrich (1982) proved that an inversive semiring $S$ is a subdirect product of a distributive lattice and a ring if and only if $S$ satisfies certain conditions. The aim of this paper is to obtain a generalized version of this result. The main purpose of this paper however, is to investigate, what new necessary and sufficient conditions need we impose on an inversive semiring, so that, in its aforesaid representation as a subdirect product, the "ring" involved can be gradually enriched to a "field." Finally, we provide a construction of full $E$-inversive semirings, which are subdirect products of a semilattice and a ring.
\end{abstract}

2000 Mathematics Subject Classification. 16Y60.

1. Introduction. In this paper, by a semiring we mean a nonempty set $S$ together with two binary operations, "+" and "." (usually denoted by juxtaposition) such that, $(S,+)$ is a commutative semigroup and $(S, \cdot)$ is a semigroup which are connected by ring-like distributivity. In particular, if $x+x=x \cdot x$ for all $x \in S$ then it is called a monosemiring [5]. An inversive semiring $S$ is a semiring in which $(S,+)$ is an inverse semigroup, that is, for each $a \in S$ there is a unique element $a^{\prime} \in S$ such that $a+$ $a^{\prime}+a=a$ and $a^{\prime}+a+a^{\prime}=a^{\prime}$. It is well known [3] that for any $a, b \in S,(a b)^{\prime}=$ $a^{\prime} b=a b^{\prime},\left(a^{\prime}\right)^{\prime}=a$, and $(a+b)^{\prime}=a^{\prime}+b^{\prime}$. Note that for an inversive semiring $(S,+, \cdot)$ the semigroup $(S,+)$ is a strong semilattice of abelian groups. A semiring $S$ is called an idempotent semiring if $x+x=x \cdot x=x$ for all $x \in S$. We denote by $E^{+}(S)$ and $E^{\cdot}(S)$, the set of all additive and multiplicative idempotents of $S$, respectively. We point out that, with respect to the operations of $(S,+, \cdot), E^{+}(S)$ is a subsemiring of $S$ which is also an ideal of $S$. A semiring $S$ is called $E$-inversive, if for every $a \in S$, there exists $x \in S$ such that $a+x \in E^{+}(S)$. A $k$-ideal $I$ of a semiring $S$ is an ideal of $S$ such that if $a \in I, x \in S$, and $a+x \in I$, then $x \in I$. A subsemiring $H$ of the direct product of two semiring $S$ and $T$ is called a subdirect product of $S$ and $T$ if the two projection mappings $\pi_{1}: H \rightarrow S$ given by $\pi_{1}(s, t)=s$ and $\pi_{2}: H \rightarrow T$ given by $\pi_{2}(s, t)=t$ are surjective. A semiring $R$ which is isomorphic to a subdirect product $H$ of $S$ and $T$ is also called a subdirect product of $S$ and $T$. In the present paper, the structure of an inversive semiring is analyzed and a generalization of a result by Bandelt and Petrich [1] is obtained. Then some necessary and sufficient conditions for an inversive semiring to be a subdirect product of different special classes of semirings are furnished. Finally, a particular construction of an $E$-inversive semiring as a subdirect product is given.

2. Subdirect products. Towards the generalization of the result by Bandelt and Petrich [1] we first prove the following lemma. 
LEMMA 2.1. Let $S$ be an inversive semiring. We define

$$
\sigma=\{(a, b) \in S \times S: a+e=b+e \text { for some } e \in E+(S)\} .
$$

Then $\sigma$ is the least ring congruence on $S$.

Proof. Straightforward.

THEOREM 2.2. An inversive semiring $S$ is a subdirect product of a ring and an additively idempotent semiring if and only if $E^{+}(S)$ is a $k$-ideal of $S$.

Proof. Let $S$ be a subdirect product of a ring $R$ and an additively idempotent semiring $T$. Then $E^{+}(S)=\left(\left\{O_{R}\right\} \times T\right) \cap S$. Let $a \in S$ be such that $a+e=f$ for some $e, f \in E^{+}(S)$. Then $e=\left(O_{R}, t_{1}\right)$ and $f=\left(O_{R}, t_{2}\right)$ for some $t_{1}, t_{2} \in T$. Then clearly $a \in\left(\left\{O_{R}\right\} \times T\right) \cap S=E^{+}(S)$ and hence $E^{+}(S)$ is a $k$-ideal of $S$. Conversely, let $E^{+}(S)$ be a $k$-ideal of $S$. We define $\psi: S \longrightarrow\left(S / \sigma \times E^{+}(S)\right)$ by $\psi(a)=\left(a \sigma, a+a^{\prime}\right)$ where $\sigma$ is as defined in Lemma 2.1. Through a routine calculation it can be shown that $\psi$ is a well defined homomorphism and the corresponding projection mappings are surjective. Also $\psi$ is injective. Indeed, let $\psi(a)=\psi(b)$ so that $a \sigma=b \sigma$ and $a+a^{\prime}=b+b^{\prime}$ for $a, b \in S$. Then $a+e=b+e$ for some $e \in E^{+}(S)$ so that $a+b^{\prime}+e=b+b^{\prime}+e \in E^{+}(S)$ whence $a+b^{\prime} \in E^{+}(S)$ as $E^{+}(S)$ is a $k$-ideal. Hence $a+b^{\prime}=a^{\prime}+b$ and also $a+b^{\prime}=(a+$ $\left.b^{\prime}\right)+\left(a^{\prime}+b\right)=a+a^{\prime}+b+b^{\prime}=b+b^{\prime}$ so that, $a=a+a^{\prime}+a=a+b^{\prime}+b=b+b^{\prime}+b=b$, whence $\psi$ is a monomorphism. It then follows that $S$ is a subdirect product of the ring $S / \sigma$ and the additively idempotent semiring $E^{+}(S)$.

COROLLARY 2.3 [1]. An inversive semiring $S$ is a subdirect product of a distributive lattice and $a$ ring if and only if $S$ satisfies the following conditions:

$\mathrm{A}(1) \quad a\left(a+a^{\prime}\right)=a+a^{\prime}$,

$\mathrm{A}(2) \quad a\left(b+b^{\prime}\right)=\left(b+b^{\prime}\right) a$,

$\mathrm{A}(3) \quad a+\left(a+a^{\prime}\right) b=a$ for all $a, b \in S$, and

$\mathrm{A}(4) \quad a \in S, a+b=b$ for some $b \in S$ implies $a+a=a$.

Proof. It is easy to verify that $E^{+}(S)$ is a distributive lattice with respect to the operations of $(S,+, \cdot)$ if and only if $S$ satisfies $\mathrm{A}(1), \mathrm{A}(2)$, and $\mathrm{A}(3)$. Indeed, let $E^{+}(S)$ be a distributive lattice with respect to the operations of $(S,+, \cdot)$. We first prove that, for all $a, b \in S,\left(a+a^{\prime}\right)\left(b+b^{\prime}\right)=a\left(b+b^{\prime}\right)=\left(a+a^{\prime}\right) b$. In fact, $\left(a+a^{\prime}\right)\left(b+b^{\prime}\right)=$ $a b+a^{\prime} b+a b^{\prime}+a^{\prime} b^{\prime}=a b+(a b)^{\prime}+(a b)^{\prime}+\left((a b)^{\prime}\right)^{\prime}=\left(a b+(a b)^{\prime}\right)+\left(a b+(a b)^{\prime}\right)=$ $a b+(a b)^{\prime}=a b+a^{\prime} b=\left(a+a^{\prime}\right) b$. Again, $\left(a+a^{\prime}\right)\left(b+b^{\prime}\right)=a b+(a b)^{\prime}=a b+a b^{\prime}=$ $a\left(b+b^{\prime}\right)$. Now we see that, $a+a^{\prime}=\left(a+a^{\prime}\right)\left(a+a^{\prime}\right)\left(\right.$ since $\left.a+a^{\prime} \in E^{+}(S)\right)=a\left(a+a^{\prime}\right)$; $a\left(b+b^{\prime}\right)=\left(a+a^{\prime}\right)\left(b+b^{\prime}\right)=\left(b+b^{\prime}\right)\left(a+a^{\prime}\right)=\left(b+b^{\prime}\right) a$; and, $\left(a+a^{\prime}\right)+\left(a+a^{\prime}\right) b=$ $\left(a+a^{\prime}\right)+\left(a+a^{\prime}\right)\left(b+b^{\prime}\right)=\left(a+a^{\prime}\right)$ which gives $a+a^{\prime}+a+\left(a+a^{\prime}\right) b=a+a^{\prime}+a$, that is, $a+\left(a+a^{\prime}\right) b=a$; for all $a, b \in S$. Conversely, let conditions $\mathrm{A}(1), \mathrm{A}(2)$, and $\mathrm{A}(3)$ hold for $S$. We point out that, for any $e \in E^{+}(S), e=e^{\prime}$; whence we have $e^{2}=e e=$ $e(e+e)=e\left(e+e^{\prime}\right)=e+e^{\prime}=e+e=e$. Again, $e f=e(f+f)=e\left(f+f^{\prime}\right)=\left(f+f^{\prime}\right) e=$ $(f+f) e=f e$ for all $e, f \in E^{+}(S)$. Also, $e+e f=e+(e+e) f=e+\left(e+e^{\prime}\right) f=e$ and $e(e+f)=e e+e f=e+e f=e$ for all $e, f \in E^{+}(S)$. Consequently, $E^{+}(S)$ is a distributive lattice with respect to the operations of $(S,+, \cdot)$. Further, $E^{+}(S)$ is a $k$-ideal of $S$ if and only if $S$ satisfies A(4). Indeed, let $E^{+}(S)$ be a $k$-ideal of $S$ and let $a+b=b$ for some 
$a, b \in S$. Then, $a+b+b^{\prime}=b+b^{\prime}$ implies $a+\left(b+b^{\prime}\right) \in E^{+}(S)$ as $b+b^{\prime} \in E^{+}(S)$, whence $a \in E^{+}(S)$ as $E^{+}(S)$ is a $k$-ideal of $S$. Conversely, let condition A(4) hold for $S$. Let $a+e=f$ for some $e, f \in E^{+}(S)$, and $a \in S$. Since we see, $a+e+e+f=e+f+f$ gives $a+e+f=e+f$, by A(4) we have $a+a=a$, that is, $a \in E^{+}(S)$ proving that $E^{+}(S)$ is a $k$-ideal of $S$. Hence the corollary follows by Theorem 2.2.

The following corollaries occur immediately.

COROLlaRY 2.4. An inversive semiring $S$ is a subdirect product of an idempotent semiring and $a$ ring if and only if $S$ satisfies $A(1)$ and $A(4)$.

COROLlaRY 2.5. An inversive semiring $S$ is a subdirect product of a commutative idempotent semiring and $a$ ring if and only if $S$ satisfies $A(1), A(2)$, and $A(4)$.

Now we establish some necessary and sufficient conditions that an inversive semiring needs to satisfy so that it can be represented as a subdirect product of certain particular classes of semirings and rings. Let $S$ be a subdirect product of an additively idempotent semiring $T$ and a ring $R$. It can be easily verified that if $S$ is an inversive semiring, then $(a, r) \in S$ implies $(a,-r) \in S$ for every $a \in E^{+}(T), r \in R$ and conversely. We now prove the following.

THEOREM 2.6. An inversive semiring $S$ is a subdirect product of a commutative idempotent semiring $T$ and $a$ ring $R$ with identity if and only if $S$ satisfies $A(1), A(2)$, $A(4)$, and condition

$\mathrm{B}(1)$ there exists $b \in E^{\cdot}(S) \backslash E^{+}(S)$ such that

$$
a+b a=a+a b=2 a+\left(a+a^{\prime}\right)\left(b+b^{\prime}\right) \quad \forall a \in S .
$$

Proof. By Corollary 2.5, $S$ is a subdirect product of a commutative idempotent semiring $T$ and a ring $R$ if and only if $S$ satisfies $\mathrm{A}(1), \mathrm{A}(2)$, and $\mathrm{A}(4)$. We show that $R$ is a ring with identity 1 if and only if $S$ satisfies $\mathrm{B}(1)$.

Suppose that $R$ has an identity 1 . Let $a \in S$; then $a=(\alpha, x)$ for some $\alpha \in T$ and $x \in$ $R$. Then $a^{\prime}=(\alpha,-x) \in S$. Now, since $1 \in R$, there exists some $\beta \in T$ such that $(\beta, 1) \in$ $S$; let $b=(\beta, 1) \in E^{\cdot}(S) \backslash E^{+}(S)$. Now, $a+a b=(\alpha, x)+(\alpha, x)(\beta, 1)=(\alpha+\alpha \beta, 2 x)$; $a+b a=(\alpha, x)+(\beta, 1)(\alpha, x)=(\alpha+\alpha \beta, 2 x)$; and $2 a+\left(a+a^{\prime}\right)\left(b+b^{\prime}\right)=2(\alpha, x)+$ $\left(\alpha, 0_{R}\right)\left(\beta, 0_{R}\right)=(\alpha+\alpha \beta, 2 x)$, whence $a+a b=a+b a=2 a+\left(a+a^{\prime}\right)\left(b+b^{\prime}\right)$ which is precisely $\mathrm{B}(1)$.

Conversely, let the condition $\mathrm{B}(1)$ hold. Let $x \in R$ be arbitrary; then $a=(\alpha, x) \in S$ for some $\alpha \in T$. By $\mathrm{B}(1)$ there exists $b=(\beta, y) \in S$ (say) such that $(\beta, y) \in E^{\cdot}(S) \backslash E^{+}(S)$ and $(\alpha, x)+(\alpha, x)(\beta, y)=2(\alpha, x)+\left(\alpha, 0_{R}\right)\left(\beta, 0_{R}\right)$, that is, $(\alpha+\alpha \beta, x+x y)=(\alpha+$ $\alpha \beta, 2 x)$ whence $x+x y=2 x$, that is, $x y=x$. Similarly, it can be shown that $y x=x$. Combining these two we get $x y=x=y x$. It follows that $y$ is the identity of $R$.

THEOREM 2.7. An inversive semiring $S$ is a subdirect product of a commutative idempotent semiring $T$ and a regular ring $R$ if and only if $S$ satisfies $A(1), A(2), A(4)$, and condition

$\mathrm{B}(2)$ for any $a \in S$, there exists $b \in S$ such that

$$
a+a b a=2 a+\left(a+a^{\prime}\right)\left(b+b^{\prime}\right) .
$$


Proof. In view of Corollary 2.5, it is sufficient to prove that the $\operatorname{ring} R$ is regular if and only if $S$ satisfies $\mathrm{B}(2)$. Suppose that $R$ is a regular ring. Let $a \in S$; then $a=(\alpha, x)$ for some $\alpha \in T$ and $x \in R$. Then there exists $y \in R$ such that $x y x=x$. Then for some $\beta \in T,(\beta, y) \in S$; let $b=(\beta, y)$. We see that $a^{\prime}=(\alpha,-x), b^{\prime}=(\beta,-y)$. Now, $a+a b a=(\alpha, x)+(\alpha, x)(\beta, y)(\alpha, x)=(\alpha, x)+(\alpha \beta \alpha, x y x)=(\alpha+\alpha \beta, 2 x)=2(\alpha, x)+$ $\left(\alpha \beta, 0_{R}\right)=2(\alpha, x)+\left(\alpha, 0_{R}\right)\left(\beta, 0_{R}\right)=2 a+\left(a+a^{\prime}\right)\left(b+b^{\prime}\right)$ which proves $\mathrm{B}(2)$.

Conversely, let $x \in R$; then $a=(\alpha, x) \in S$ for some $\alpha \in T$. Now, by $\mathrm{B}(2)$ there exists $b=(\beta, y) \in S$, for some $\beta \in T$ and $y \in R$ such that $(\alpha, x)+(\alpha, x)(\beta, y)(\alpha, x)=$ $2(\alpha, x)+\left(\alpha, 0_{R}\right)\left(\beta, 0_{R}\right)$ whence $x+x y x=2 x$ which implies $x y x=x$, proving, thereby, the regularity of $R$.

COROLLARY 2.8. An inversive semiring $S$ is a subdirect product of a distributive lattice $D$ and a regular ring $R$ if and only if $S$ satisfies $A(1), A(2), A(4)$, and the condition $\mathrm{B}(2)^{*}$ for any $a \in S$, there exists some $b \in S$ such that $a+a b a=2 a$.

Proof. Let $S$ be an inversive semiring such that $S$ is a subdirect product of a distributive lattice $D$ and a regular ring $R$. Then from Corollary 2.3 and Theorem 2.7 we find that $S$ satisfies all the conditions $\mathrm{A}(1), \mathrm{A}(2), \mathrm{A}(3), \mathrm{A}(4), \mathrm{B}(2)$, and hence $\mathrm{B}(2)^{*}$.

Conversely, if $S$ is an inversive semiring satisfying the conditions $\mathrm{A}(1), \mathrm{A}(2), \mathrm{A}(4)$, and $\mathrm{B}(2) *$; we observe that it also satisfies $\mathrm{A}(3)$. Indeed, let $a+a^{\prime}=e$ for some $a \in S$. Then from $\mathrm{B}(2)^{*}$ we have $e+e b e=2 e=e$, whence by $\mathrm{A}(2)$ it implies $e+e e b=e$, that is, $e+e b=e\left(\right.$ by A(1)), that is, $a+a^{\prime}+\left(a+a^{\prime}\right) b=a+a^{\prime}$ which implies $a+\left(a+a^{\prime}\right) b=a$. It now suffices to show that the regularity of $R$ follows from $\mathrm{B}(2)^{* *}$. Indeed, let $x \in R$; then $a=(\alpha, x) \in S$ for some $\alpha \in D$. By $\mathrm{B}(2)^{*}$, there exists $b=(\beta, y) \in S$ for some $\beta \in D$ and $y \in R$ such that $(\alpha, x)+(\alpha, x)(\beta, y)(\alpha, x)=2(\alpha, x)$ whence $x+x y x=2 x$ and $x y x=x$ showing that $R$ is a regular ring.

THEOREM 2.9. A commutative inversive semiring $S$ is a subdirect product of a commutative idempotent semiring $T$ and an integral domain $R$ if and only if $S$ satisfies $A(1)$, $A(2), A(4), B(1)$, and the condition

$\mathrm{B}(3) a b \in E^{+}(S)$ for $a, b \in S$ implies, either $a \in E^{+}(S)$ or $b \in E^{+}(S)$.

Proof. By Corollary 2.5, a commutative inversive semiring $S$ is a subdirect product of a commutative idempotent semiring $T$ and a commutative ring $R$ if and only if $S$ satisfies $\mathrm{A}(1), \mathrm{A}(2)$, and $\mathrm{A}(4)$. By Theorem 2.6, $R$ is a ring with identity if and only if $S$ also satisfies $\mathrm{B}(1)$. We now show that $R$ does not admit divisors of zero if and only if $S$ satisfies B(3). Suppose that $R$ does not admit divisors of zero. Let $a \in S$ such that $a=(\alpha, x)$ for some $\alpha \in T, x \in R$, and $b \in S$ such that $b=(\beta, y)$ for some $\beta \in T$ and $y \in R$ where $a b \in E^{+}(S)$. This implies $(\alpha, x)(\beta, y) \in E^{+}(S)$, that is, $(\alpha \beta, x y)=\left(\gamma, 0_{R}\right)$ for some $\gamma \in T$ whence $x y=0_{R}$, that is, either $x=0_{R}$ or $y=0_{R}$, that is, $a \in E^{+}(S)$ or $b \in E^{+}(S)$ which is precisely $\mathrm{B}(3)$.

Conversely, let $x, y \in R$ such that $x y=0_{R}$; then $a=(\alpha, x) \in S$ and $b=(\beta, y) \in S$ for some $\alpha, \beta \in T$. Now, we see that $a b=(\alpha, x)(\beta, y)=\left(\alpha \beta, 0_{R}\right) \in E^{+}(S)$ whence from $\mathrm{B}(3)$ we get, either $(\alpha, x) \in E^{+}(S)$ or $(\beta, y) \in E^{+}(S)$, that is, either $x=0_{R}$ or $y=0_{R}$. Consequently, $R$ is free from divisors of zero.

Combining Theorems 2.7 and 2.9 we get the following result. 
THEOREM 2.10. A commutative inversive semiring $S$ is a subdirect product of a commutative idempotent semiring $T$ and a field $F$ if and only if $S$ satisfies the conditions $A(1), A(2), A(4), B(1), B(2)$, and $B(3)$.

Now, we establish a certain class of $E$-inversive semiring as a particular type of subdirect product of a monosemiring and a ring. In the light of Mitsch [4], we give the following definitions.

DEFINITION 2.11. A subdirect product $T$ of a semiring $S$ and a $\operatorname{ring} R$ is called full, if $\left(e, 0_{R}\right) \in T$ for all $e \in E^{+}(S)$.

DefinITION 2.12 [2]. A subdirect product $T$ of a semiring $S$ and a ring $R$ is called inversive, if $(e, r) \in T$ implies $(e,-r) \in T$ for every $e \in E^{+}(S), r \in R$.

Clearly, a subdirect product $S$ of a distributive lattice and a ring is inversive if and only if $S$ is an inversive semiring; whereas any subdirect product of a distributive lattice and a ring is full.

THEOREM 2.13. Let $Y$ be a semilattice and $R$ a ring. Suppose that there is a mapping $f$ from $(Y, \leq)$ into the semilattice $(u(R), \subseteq)$ of all subsemirings with $0_{R}$, ordered by inclusion, which is order inverting and satisfies $R=\bigcup_{\alpha \in Y} \alpha f$. Then

$$
S=\{(\alpha, a) \in Y \times R \mid a \in \alpha f\},
$$

with the operations defined by

$$
(\alpha, a) \oplus(\beta, b)=(\alpha \beta, a+b), \quad(\alpha, a) \cdot(\beta, b)=(\alpha \beta, a b),
$$

is an E-inversive semiring, which is a full subdirect product of the commutative idempotent monosemiring $(Y,+, \cdot)$ and the ring $R$. Conversely, every such semiring can be constructed in this way.

Proof. The set $S$ is a semiring with the aforesaid operations. Indeed, $(\alpha, a),(\beta, b) \in$ $S$ imply that $a \in \alpha f \subseteq(\alpha \beta) f, b \in \beta f \subseteq(\alpha \beta) f$ so that $a b, a+b \in(\alpha \beta) f$ and hence $(\alpha \beta, a b),(\alpha \beta, a+b) \in S$. Further, it is easy to see that,

$$
(\alpha, a) \cdot[(\beta, b) \oplus(\gamma, c)]=(\alpha, a) \cdot(\beta, b) \oplus(\alpha, a) \cdot(\gamma, c) .
$$

Now from the condition, $\bigcup_{\alpha \in Y} \alpha f=R$, we see that, $S$ is $E$-inversive. In fact, for any $(\alpha, a) \in S$ we have for $(\beta,-a) \in S$ such that $(\alpha, a) \oplus(\beta,-a)=\left(\alpha \beta, 0_{R}\right) \in E^{+}(S)$. Again, since $\alpha f \neq \phi$ for all $\alpha \in Y$ and since $\bigcup_{\alpha \in Y} \alpha f=R$, we see $S$ is a subdirect product of the monosemiring $Y$ and the ring $R$. It is a full subdirect product, since every $\alpha f \in u(R)$ is a subsemiring of $R$ containing $0_{R}$, hence $0_{R} \in \alpha f$ and so $\left(\alpha, 0_{R}\right) \in S$, for every $\alpha \in Y$.

Conversely, let $S$ be a full subdirect product of a commutative idempotent monosemiring $Y$ and a ring $R$. We define $\alpha f=\{a \in R \mid(\alpha, a) \in S\}$ for every $\alpha \in Y$. It is a routine matter to check that $\alpha f$ as defined above is a subsemiring of $R$ containing $0_{R}$. So the mapping $f: Y \rightarrow u(R)$ given by $\alpha \mapsto \alpha f$ is defined. We point out that the commutative idempotent monosemiring $Y$ can be seen as a semilattice $(Y, \leq)$ by defining the partial order " $\leq$ " by $\alpha \leq \beta$ if and only if $\alpha=\alpha \beta$ for $\alpha, \beta \in Y$. If $\alpha \leq \beta$ in $Y$ 
and $x \in \beta f$, then $\alpha=\alpha \beta$ and $(\beta, x) \in S$, hence $(\alpha, x)=(\alpha \beta, x)=\left(\alpha, 0_{R}\right) \oplus(\beta, x) \in S$ by hypothesis and so $x \in \alpha f$ which implies $\beta f \subseteq \alpha f$, that is, $f$ is order inverting. Since $S$ is a subdirect product of $Y$ and $R, \bigcup_{\alpha \in Y} \alpha f=R$ is satisfied. In fact, $\bigcup_{\alpha \in Y} \alpha f \subseteq R$ holds trivially. To check the reverse inclusion let $r \in R$ be arbitrary. Since $S$ is a subdirect product of $Y$ and $R$, there exists some $\gamma \in Y$, such that $(\gamma, r) \in S$, that is, $r \in \gamma f$ and $r \in \bigcup_{\alpha \in Y} \alpha f$. Now it is evident that $S=\{(\alpha, a) \in Y \times R \mid a \in \alpha f\}$.

COROLlary 2.14. Let $Y$ be a semilattice and $R$ a ring. Suppose that there is a mapping $f$ from $(Y, \leq)$ into the semilattice $(U(R), \subseteq)$ of all subrings, ordered by inclusion, which is order inverting and satisfies $R=\bigcup_{\alpha \in Y} \alpha f$. Then

$$
S=\{(\alpha, a) \in Y \times R \mid a \in \alpha f\},
$$

with the operations defined by

$$
(\alpha, a) \oplus(\beta, b)=(\alpha \beta, a+b), \quad(\alpha, a) \cdot(\beta, b)=(\alpha \beta, a b),
$$

is a semiring, which is an inversive subdirect product of the commutative idempotent monosemiring $(Y,+, \cdot)$ and the ring $R$. Conversely, every such semiring can be constructed in this way.

Proof. In view of Theorem 2.13 it is sufficient to prove that $S$ is inversive if and only if $\alpha f$ is a subring of $R$ for each $\alpha \in Y$. Let $\alpha f$ be a subring for each $\alpha \in Y$. Now, for some $a \in R,(\alpha, a) \in S$ implies $a \in \alpha f$ whence we must have $-a \in \alpha f$ and hence $(\alpha,-a) \in S$ showing that $S$ is inversive. The converse is straightforward.

THEOREM 2.15. Let $Y$ be a distributive lattice and $R$ a ring. Suppose that there is a mapping $f$ from $(Y, \leq)$ into the lattice $(u(R), \subseteq)$ of all subsemirings with $0_{R}$, ordered by inclusion, such that

(i) $f$ is order inverting,

(ii) $\alpha f+\beta f \subseteq(\alpha+\beta) f$ for all $\alpha, \beta \in Y$,

(iii) $R=\bigcup_{\alpha \in Y} \alpha f$.

Then

$$
S=\{(\alpha, a) \in Y \times R \mid a \in \alpha f\},
$$

with the operations defined by

$$
(\alpha, a) \oplus(\beta, b)=(\alpha+\beta, a+b), \quad(\alpha, a) \cdot(\beta, b)=(\alpha \beta, a b),
$$

is an E-inversive semiring which is a subdirect product of the distributive lattice $Y$ and the ring $R$. Conversely, each such semiring can be constructed in this way.

Proof. The semiring $S$ is closed under the aforesaid operation of "addition" $\oplus$. Indeed, $(\alpha, a),(\beta, b) \in S$ imply that $a \in \alpha f, b \in \beta f$, so that $a+b \in \alpha f+\beta f \subseteq(\alpha+\beta) f$ whence $(\alpha+\beta, a+b) \in S$. The rest of the proof of this part follows from Theorem 2.13.

Conversely, let $S$ be a subdirect product of a distributive lattice $Y$ and a ring $R$. We define $\alpha f=\{a \in R \mid(\alpha, a) \in S\}$ for every $\alpha \in Y$. Let $x \in \alpha f$ and $y \in \beta f$ for some $\alpha, \beta \in Y$; then $(\alpha, x),(\beta, y) \in S$ whence $(\alpha+\beta, x+y) \in S$ so that we have $x+y \in$ $(\alpha+\beta) f$ proving thereby $\alpha f+\beta f \subseteq(\alpha+\beta) f$. The rest of the proof is similar to Theorem 2.13 and hence is omitted. 
Acknowledgement. The author is grateful to Dr M. K. Sen of Department of Pure Mathematics, University of Calcutta, for suggesting this problem. He also expresses his heartfelt thanks to the learned referee for his meticulous reading of the paper and his kind suggestions, which has definitely improved the final version of this paper.

\section{REFERENCES}

[1] H.-J. Bandelt and M. Petrich, Subdirect products of rings and distributive lattices, Proc. Edinburgh Math. Soc. (2) 25 (1982), no. 2, 155-171. MR 84g:16030. Zbl 486.16026.

[2] S. Ghosh, A characterization of semirings which are subdirect products of a distributive lattice and a ring, Semigroup Forum 59 (1999), no. 1, 106-120. Zbl 948.16036.

[3] P. H. Karvellas, Inversive semirings, J. Austral. Math. Soc. 18 (1974), 277-288. MR 51 \#3236. Zbl 301.16029.

[4] H. Mitsch, Subdirect products of E-inversive semigroups, J. Austral. Math. Soc. Ser. A 48 (1990), no. 1, 66-78. MR 91f:20066. Zbl 691.20050.

[5] F. Pastijn and A. Romanowska, Idempotent distributive semirings. I, Acta Sci. Math. (Szeged) 44 (1982), no. 3-4, 239-253. MR 84f:16043a. Zbl 514.16026.

P. Mukhopadhyay: Department of Pure Mathematics, University of Calcutta, 35, BALlygunge Circular RoAd, CALCUTTA-700019, INDia 


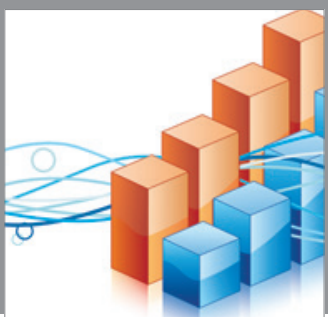

Advances in

Operations Research

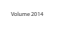

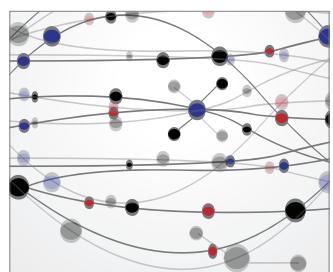

\section{The Scientific} World Journal
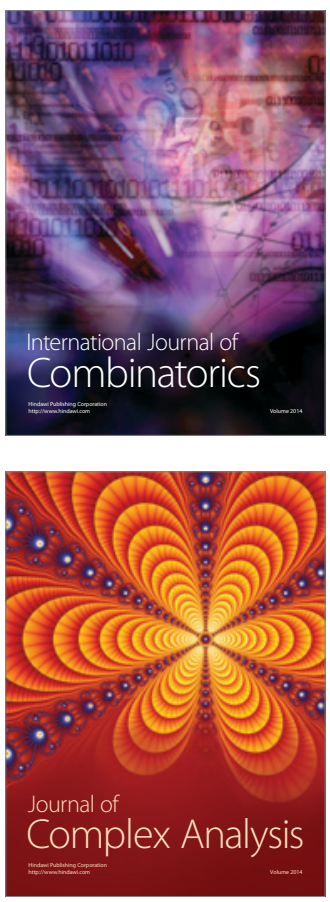

International Journal of

Mathematics and

Mathematical

Sciences
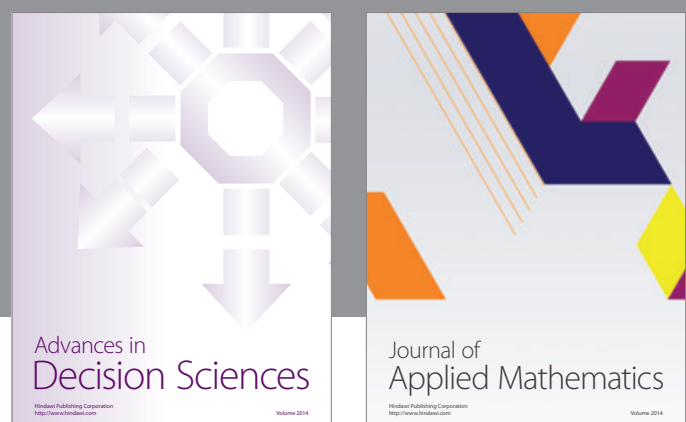

Journal of

Applied Mathematics
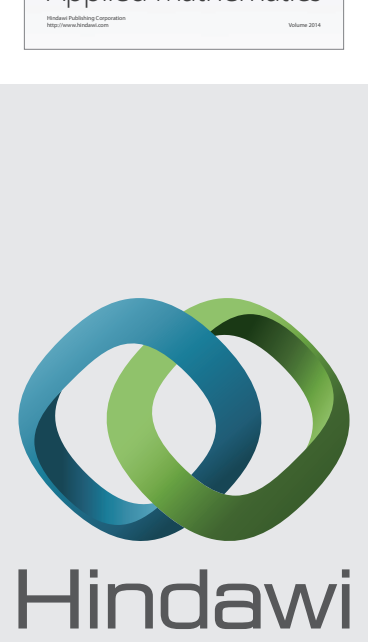

Submit your manuscripts at http://www.hindawi.com
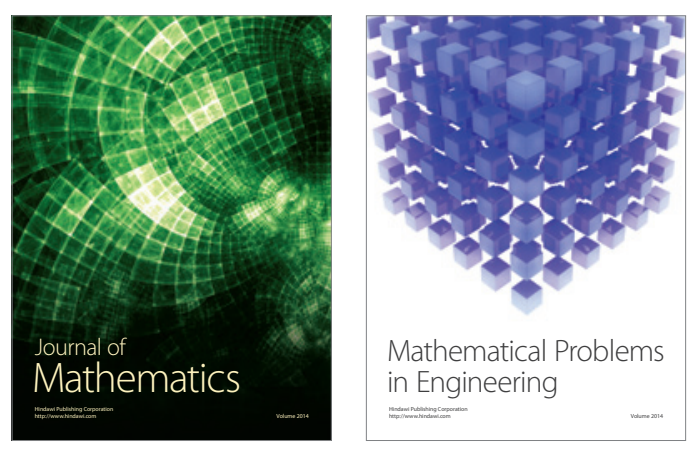

Mathematical Problems in Engineering
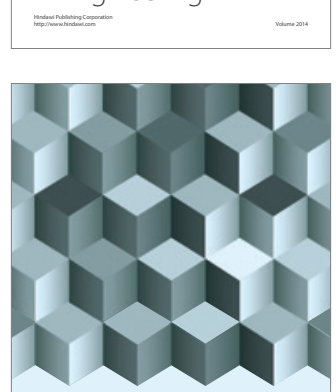

Journal of

Function Spaces
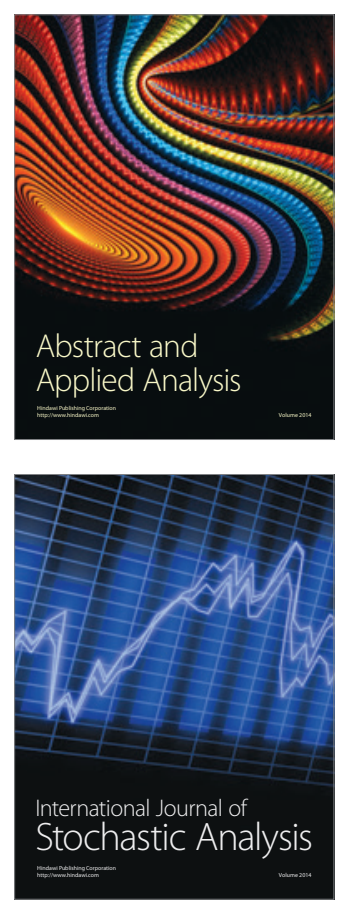

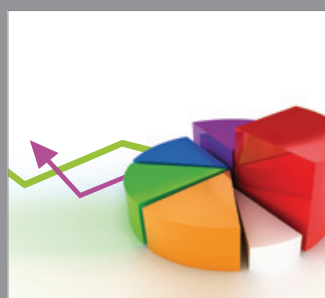

ournal of

Probability and Statistics

Promensencen
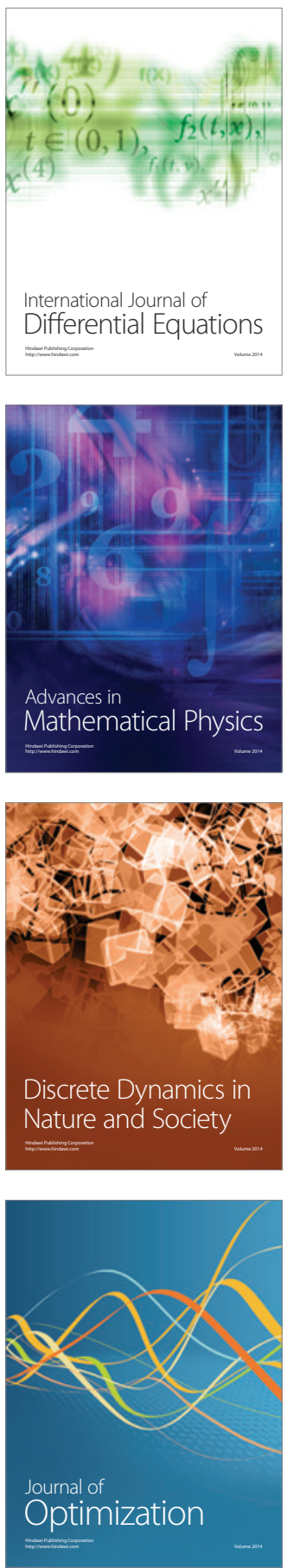